\title{
Association of the Hospitalization and in-hospital Mortality Rates with Aspartate Aminotransferase, Alanine Aminotransferase, Lactate Dehydrogenase, C-Reactive Protein, and Prothrombin Time Levels in Cancer Patients Admitted to the Emergency Department
}

\author{
(1) Sedat BíLGE, (1) Yahya Ayhan ACAR
}

Department of Emergency Medicine, University of Health Sciences, Gülhane School of Medicine, Ankara-Turkey

\begin{abstract}
OBJECTIVE
This study determined predictive risk factors for hospitalization and in-hospital mortality (IHM) in cancer patients presenting to the emergency department (ED).

METHODS

Patients diagnosed with cancer who visited the general ED were reviewed retrospectively for the period from October 01, 2016, to April 01, 2019. We recorded age, sex, triage category, and vital signs at ED admission and laboratory results of blood samples collected in the ED. Data were analyzed to evaluate the association of the abovementioned parameters with rates of hospitalization and IHM.

\section{RESULTS}

We enrolled 493 patients (males 283 [57.4\%], females 210 [42.6\%]; mean age \pm SD [range] 59.58 \pm 17.07 [18-96] years). The overall hospitalization rate was $26.4 \%$; IHM occurred in 90 (18.3\%) patients. Levels of aspartate aminotransferase (AST), alanine aminotransferase (ALT), lactate dehydrogenase (LDH), and C-reactive protein (CRP), as well as the prothrombin time (PTT) differed significantly between hospitalized and non-hospitalized ( $\mathrm{p}=0.001,0.003,0.011,0.020$, and 0.031 ; respectively) patient groups and the survivor and non-survivor ( $\mathrm{p}=<0.001,0.015,<0.001,<0.001$, and 0.005 ; respectively) hospitalized patient groups.

\section{CONCLUSION}

Vital signs, liver function tests, and coagulation parameters at ED admission could predict IHM of cancer patients. These parameters must be evaluated in oncology patients admitted to the ED.

Keywords: Cancer; coagulation; emergency department; hospitalization; mortality.

Copyright $\odot$ 2019, Turkish Society for Radiation Oncology
\end{abstract}

\section{Introduction}

Cancer is the second most prevalent cause of mortality across all leading causes of death.[1] There is an increasing incidence of malignant tumors worldwide, and a progressively higher frequency of emergency department (ED) visits by cancer patients due to various reasons.[2] Oncology patients constitute a risk group across ED patients.[3] The incidence of ED visits ranges from $7 \%$ to $12 \%$ in cancer patients, and some 
risk factors for ED visits, such as the black race, male gender, advanced age, medication non-adherence, and living alone, have been previously reported.[4] Most cancer patients need hospitalization in the last months of their lives and their frequency of ED visits increases due to inadequate provision of palliative care.[4] However, ED crowding is increasingly a problem, and cancer patients often experience longer ED waiting times due to their possible multisystem pathologies.[5] In addition to the systemic effects of malignancy, the potential for metastatic organ involvement may result in excessive use of hospital resources, such as laboratory procedures and various imaging tools, during the ED stay of cancer patients.[5]

Most ED admissions or visits of cancer patients are not due to cancer-related problems; instead, are attributable to pain, nausea, vomiting, infection, local mass effect, pleural effusion, ascites, chemotherapy/radiotherapy side effects, and hematologic or metabolic disorders.[6-8] Because patients admitted to the ED need a multidisciplinary approach and as the oncology, palliative care, and intensive care units are similarly overburdened as the ED, it is crucial for the emergency physician to be aware of the characteristics and risk factors of cancer patients for effective resource management and swiftly making a precise diagnosis.

This study was conducted to determine the risk factors for hospitalization and in-hospital mortality (IHM) in adult ED patients with cancer.

\section{Materials and Methods}

This retrospective observational study was approved by the Non-Interventional Research Ethics Committee, Gülhane School of Medicine, University of Health Sciences (approval no. 19/253, June 11, 2019). We reviewed the patient charts and records in the Hospital Data Management System to identify patients who presented to the general ED of the hospital in the period from October 1, 2016, to April 1, 2019. Patients with a diagnosis of any type of cancer were eligible for study inclusion. We excluded patients younger than 18 years, with missing data, of patients referred to an external treatment center (Fig. 1).

For the eligible cancer patients included in this study, we identified and recorded the following information: age, gender, triage category, and vital signs at admission; test results of complete blood count, routine biochemistry, coagulation panels, cardiac panels, and blood gas parameters obtained at ED visit; and whether the patients arrived at the ED by ambulance

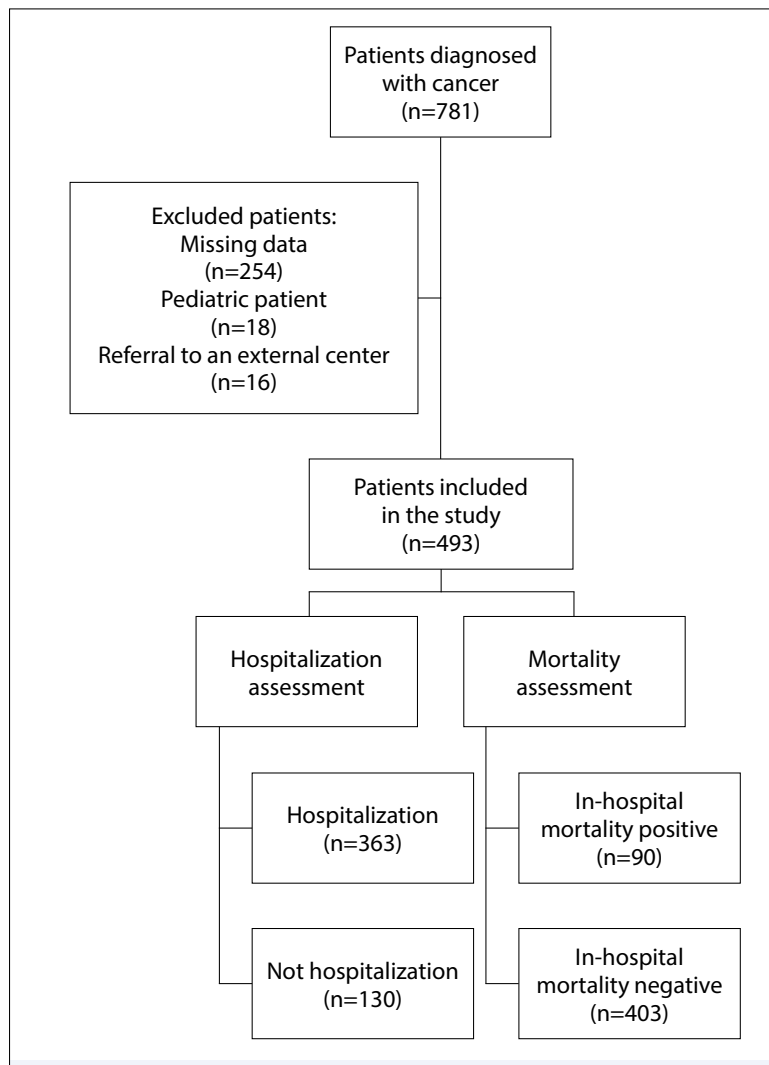

Fig. 1. Flowchart of the study.

or underwent cardiopulmonary resuscitation (CPR) in ED. Triage categories were classified according to the Turkish Ministry of Health triage system, which comprises different codes to specify priority to ED patients: a black code indicates patients with the cardiopulmonary arrest at the admission; a red code indicates patients who need emergent resuscitative efforts for survival; a yellow code specifies patients who require close observation, multiple resources (e.g., laboratory, radiological, intravenous intervention), having a high-risk medical history (e.g., diabetes, pregnancy, prior heart attack), or admitted with a critical complaint (e.g., chest pain, severe abdominal pain), as they may be stable but at the risk of immediate danger; and the green code is for patients with stable vital signs (such as pulse rate, respiratory rate, body temperature, oxygen saturation, arterial blood pressure), without any critical complaints, and are not expected to develop a dangerous medical condition during their ED stay.[9]

We stratified our study participants into hospitalized and non-hospitalized patient subgroups, as well as a survivor (no-IHM) and non-survivor hospitalized patient (IHM) groups, based on mortality from the time of hospitalization until discharge. 
Statistical Analysis: Demographic data were presented as frequencies and percentages. Normally distributed continuous variables are presented as mean and standard deviation, whereas non-normally distributed variables were presented as the median and interquartile range (IQR). The chi-square test was used for the comparison of two groups concerning categorical variables. The Mann-Whitney $U$ test and Student's t-test were used for pairwise comparisons of continuous data that did not or did conform to a normal distribution, respectively. All statistical analyses were conducted in SPSS 18.0 software. A p-value of $<0.05$ was considered statistically significant.

Power Analysis: For an alpha value of 0.05, 80\% power, enrollment ratio of 1 , and an expected hospitalization rate of $50 \%$, we calculated that this study should include 338 patients.

\section{Results}

We identified 781 patients, excluded 288 patients, and included 493 patients (males 283 [57.4\%], females 210 [42.6\%]; mean age \pm SD [range] 59.58 \pm 17.07 [18-96] years) in this study, a study flowchart presents patient disposition (Fig. 1). There were no significant betweengroup differences in age and sex between the hospitalized and non-hospitalized patient groups and the noIHM and IHM groups (Table 1). The mean length of ED stay was 189.50 (IQR: 91-321.25) minutes.

The commonest presenting complaints were pain $(\mathrm{n}=187,37.9 \%)$, fever $(\mathrm{n}=71,14.4 \%)$, nausea/vomiting $(\mathrm{n}=56,11.4 \%)$, and dyspnea $(\mathrm{n}=55,11.2 \%)$. The presenting complaints were not significantly different in the hospitalization and IHM groups (Table 2). Moreover, $327(66.3 \%)$ arrived at the ED by ambulance and $166(33.7 \%)$ by their vehicles. Stratification of triage codes indicated $138(28.0 \%)$ red triage, $350(71.0 \%)$ yellow triage, and 5 (1.0\%) black triage code patients; no cancer patients were assigned a green triage code. We detected a statistically significant relationship between the triage codes and hospitalization rates ( $\mathrm{p}=0.001$, chi-square test), but this was not related to IHM ( $\mathrm{p}=0.431$, chi-square test).

Among the patients who visited the ER, 130 (26.4\%) were hospitalized and $363(73.6 \%)$ were discharged; moreover, $16(3.2 \%)$ patients were transferred from the ED to another hospital. The hospitalized patients were further stratified by hospitalization in the medical oncology clinic ( $\mathrm{n}=182,36.9 \%)$, intensive care unit $(\mathrm{n}=18$, $3.7 \%)$, palliative care clinic $(\mathrm{n}=2,0.4 \%)$, and other clinical departments $(n=202,59.0 \%)$. Sixteen $(3.2 \%)$ patients underwent cardiopulmonary resuscitation

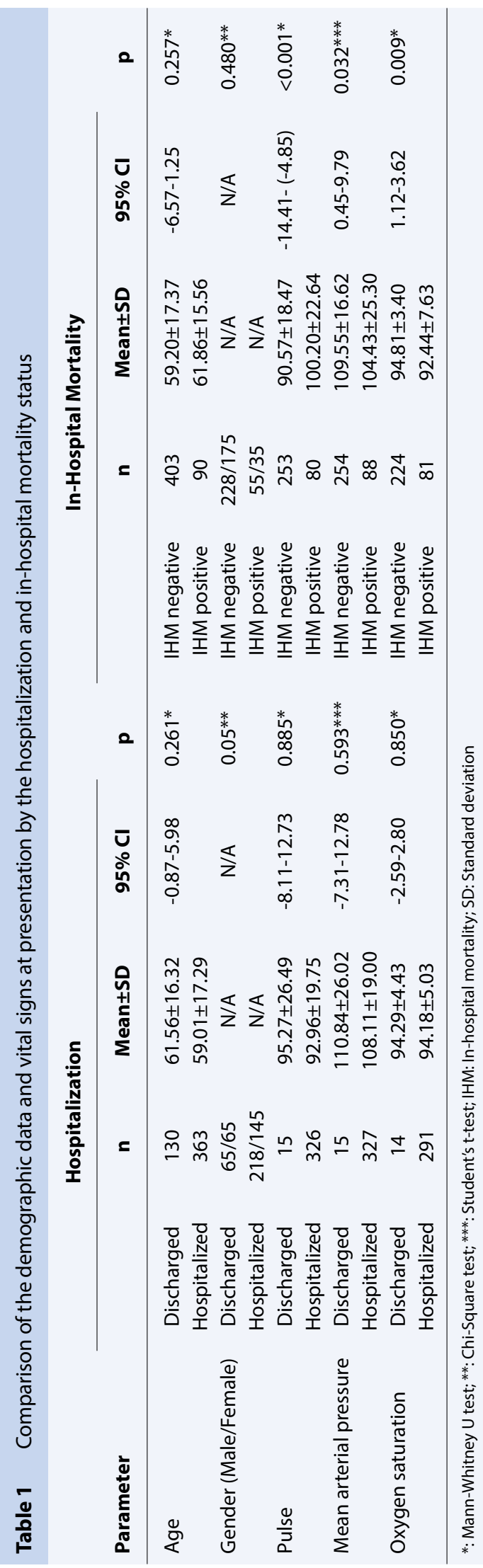


Table 2 Distribution of the patients' complaints

\begin{tabular}{lccc} 
Symptoms & $\mathbf{n}(\%)$ & $\begin{array}{c}\text { Hospitalized/ } \\
\text { not hospitalized }\end{array}$ & $\begin{array}{c}\text { With IHM/ } \\
\text { without IHM }\end{array}$ \\
\hline Fever & $71(14.4)$ & $52 / 19$ & $12 / 59$ \\
Nausea and vomiting & $56(11.4)$ & $37 / 19$ & $8 / 48$ \\
Shortness of breath & $55(11.2)$ & $41 / 14$ & $20 / 35$ \\
Weakness, fatigue & $18(3.7)$ & $13 / 5$ & $2 / 16$ \\
Epilepsy, hemiplegia, neurological & $15(3.0)$ & $12 / 3$ & $4 / 11$ \\
Bleeding & $13(2.6)$ & $11 / 2$ & $1 / 12$ \\
Syncope & $13(2.6)$ & $12 / 1$ & $3 / 10$ \\
Renal insufficiency & $10(2.0)$ & $5 / 5$ & $1 / 9$ \\
Palpitations, hypotension & $10(2.0)$ & $6 / 4$ & $2 / 8$ \\
Diarrhea, constipation & $6(1.2)$ & $5 / 1$ & $2 / 4$ \\
Other & $39(7.9)$ & $29 / 10$ & $4 / 35$ \\
Pain & $187(37.9)$ & $140 / 47$ & $31 / 156$ \\
Total & $493(100)$ & $363 / 130$ & $90 / 403$ \\
- Acute pain & $120(24.3)$ & $87 / 33$ & $20 / 100$ \\
• Abdominal pain & $41(8.3)$ & $36 / 5$ & $9 / 32$ \\
• Chest pain & $15(3.0)$ & $8 / 7$ & 0.582 \\
• Low back/musculoskeletal pain & $7(1.4)$ & $6 / 1$ & 0.087 \\
• Headache & $4(0.8)$ & $3 / 1$ & $2 / 15$ \\
\hline
\end{tabular}

*: Chi-square test; IHM: In-hospital mortality

during the ED stay, and IHM occurred in 90 (18.3\%) patients. The identified infections were pneumonia $(n=22,4.5 \%)$, urinary tract infections $(n=16,3.2 \%)$, upper respiratory tract infections $(n=6,1.2 \%)$, cellulitis $(\mathrm{n}=6,1.2 \%)$, biliary tract infections (cholecystitis; $\mathrm{n}=1$, $0.2 \%)$, and pancreatitis $(\mathrm{n}=1,0.2 \%)$.

We detected significant between-group differences in the levels of hemoglobin ( $\mathrm{Hb})$, aspartate aminotransferase (AST), alanine aminotransferase (ALT), lactate dehydrogenase (LDH), C-reactive protein (CRP), lactate, and prothrombin time (PTT) (Tables 1-4) in the hospitalized and non-hospitalized groups. Moreover, we found significant between-group differences in the pulse rate, diastolic blood pressure, oxygen saturation $\left(\mathrm{SaO}_{2}\right)$, white blood cell count (WBC), PTT, and international normalized ratio (INR), and the levels of glucose, urea, AST, ALT, LDH, CRP, PTT, troponin, and lactate (Tables 1-4) in the IHM and no-IHM groups.

\section{Discussion}

The results of the present study show that emergency physicians can predict the IHM of cancer patients via a detailed examination of the vital signs, liver function tests, and coagulation parameters at the time of ED admission.

The age and sex distribution of cancer patients admitted to the ED were consistent with the reports in the literature.[5-8] Given that cancer is commonest at the advanced age (fifth and sixth decades), our study had a distribution of cancer patients who visited the ED that was in agreement with this information. As cancer patients constitute a risk group among other patients with $\mathrm{ED}$, they are hospitalized more commonly compared to the non-cancer patients.[1,5-7] Moreover, the IHM rate among cancer patients in our study was consistent with reports in the available literature $[7,8,10,11]$, which supports the more frequent observation of high-risk triage categories and more frequent hospitalizations in our study.

Conventional cancer treatment includes chemotherapy and radiotherapy. Cancer staging determines the treatment modality and mortality in these patients but has limited value for the emergency physician. [12] This is because acute and life-threatening conditions, such as hematological disorders (e.g., neutropenia, bleeding diathesis), symptoms due to compression by primary or metastatic mass, acute pain, predisposition to thromboembolic events, as well as radiotherapy- and chemotherapy-induced immunosuppression, are primarily evaluated during ED procedures.[12] In the present study, these conditions are indicated by an increase in infective parameters, such as WBC, CRP, bleeding diathesis (caused by INR elevation), and increased levels of liver enzymes. Therefore, we recom- 


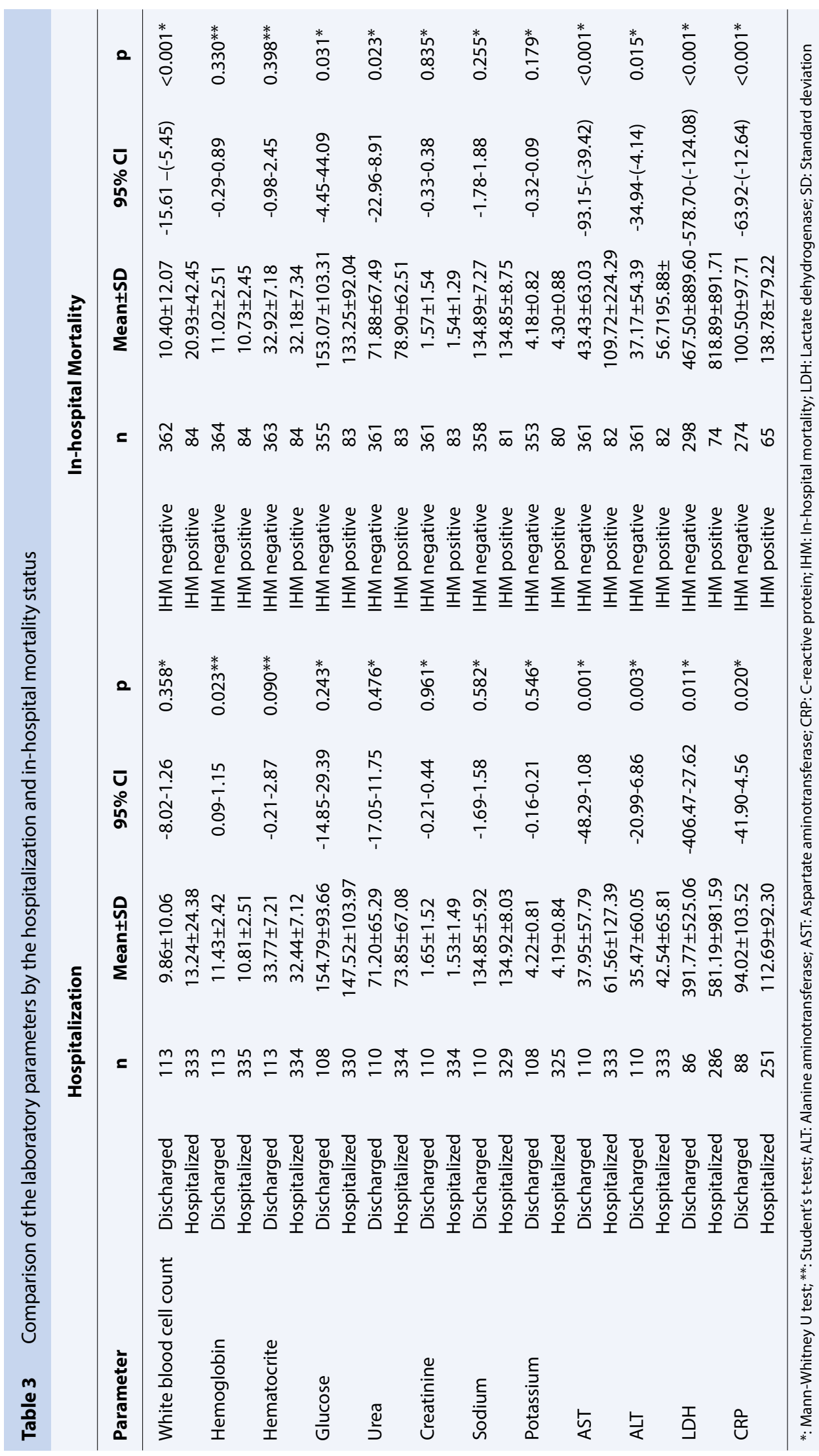




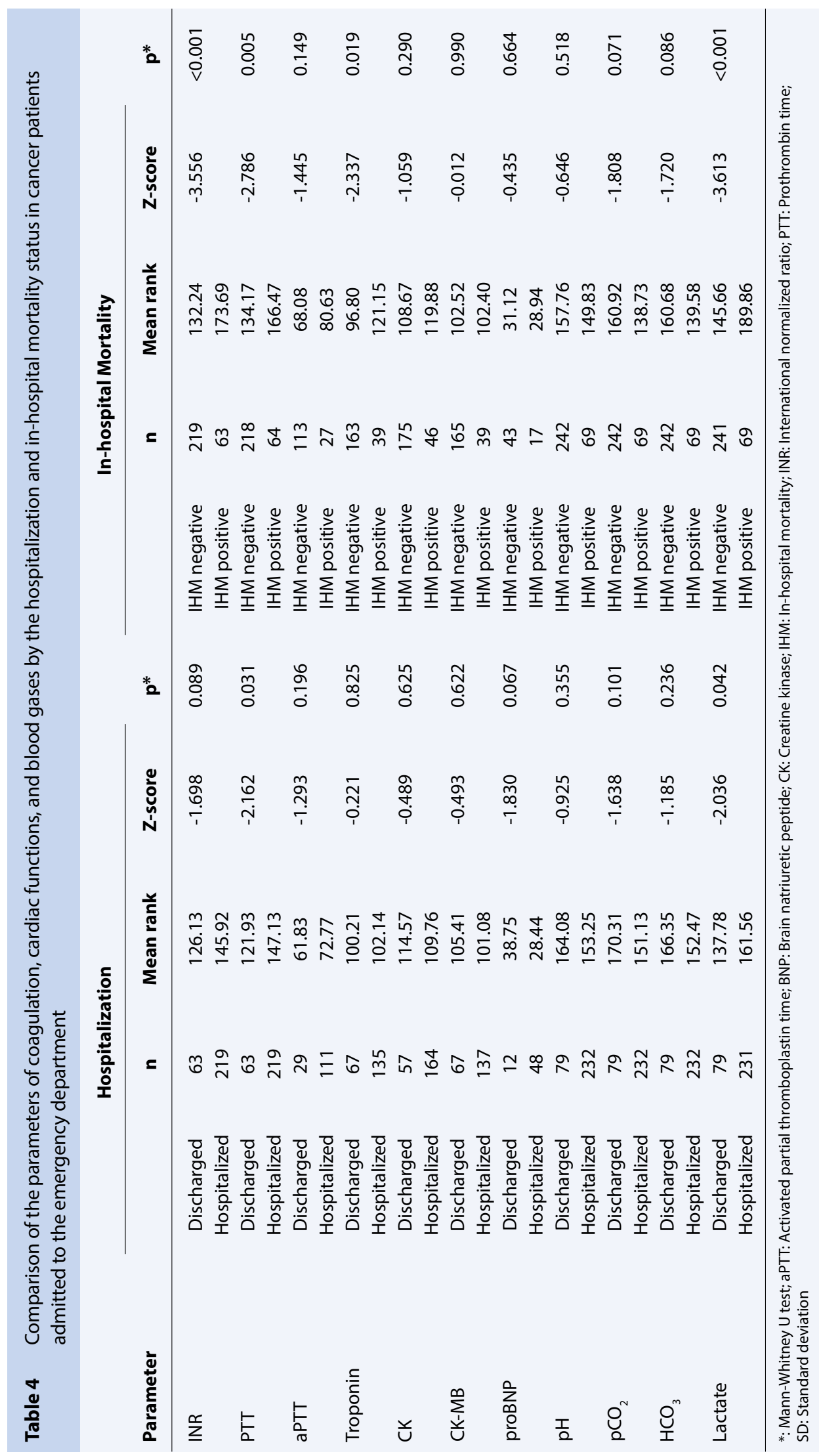


mend that emergency physicians and other clinicians should evaluate laboratory findings together with the patients' vital signs (hypotension, tachycardia and hypoxemia).

Cancer patients visited the ED mostly between 12 p.m. and 3 a.m., similar to non-cancer patients, and experienced significantly longer ED wait times than non-cancer patients. $[5,6,8,10]$ Yates et al. reported no association between the cause of hospitalization and length of stay.[8] In the present study, we found that the mean length of ED stay of cancer patients was shorter than the time reported in the literature. $[8,10]$ Notably, ED practices in Turkey are regulated by local legislation, which requires strict compliance. A decree of the Emergency Department Legislation, issued by the Republic of Turkey's Ministry of Health, stipulates patients with a defined diagnosis should be hospitalized or discharged from the ED within four hours, whereas patients without any defined diagnoses must be hospitalized or discharged from the ED within eight hours. Therefore, the short ED waiting period identified in the present study is attributable to the implementation of regulatory requirements, undertaken to reduce ER wait time in the respective hospital to prevent non-compliance to legislation and future potential sanctions.

Inflammatory processes play a role in the development and progression of cancer and infections and are reported as the commonest cause (19.4\%) of hospitalization across all identified etiological factors.[1, 8] Wulaningsih et al. reported that high levels of CRP were associated with mortality in cancer patients, and are potentially related to chronic inflammation.[13] We found increased levels of inflammatory markers (WBC counts, CRP levels) in hospitalized patients and in the IHM group. Thus, infections possibly prolong the length of hospital stay and increase the risk of mortality, and infectious processes are simultaneously involved in tumor progression.

Cancer affects the management of diabetes adversely in association with increased mortality in cancer patients. [14] In contrast, Boursi et al. reported that high blood glucose levels are associated with better prognosis in cancer patients.[15] We found low glucose levels in the IHM group, which is consistent with the findings of Boursi et al. However, the information in the literature on the value of glucose levels in predicting mortality in cancer patients is controversial, and the exact mechanism underlying these findings has not been clarified yet.

Yang et al. reported that high levels of BUN and creatinine were risk factors for mortality in septic can- cer patients.[16] We found higher urea levels of IHM patients, indicating that the association between increased urea levels and death might be secondary to the evolution of multiorgan failure. Oh et al. reported that increased ALT and GGT levels were associated with mortality in patients younger than 60 years. [17] Wulaningsih et al. reported that higher LDH levels might be associated with mortality in cancer patients due to chronic hypoxia with excessive energy use in rapidly proliferating cancer cells.[18] In concordance with these reports, we found higher levels of AST, ALT, and LDH in both hospitalized and IHM groups than non-hospitalized and non-IHM subgroups. However, there are no proposed mechanisms in the literature to precisely explain this observation. Castle et al. reported that prolonged PTT or INR values in pancreatectomy patients were associated with increased 30-day mortality rates. [19] Fischer et al. reported a close relationship between inflammation and several coagulation parameters, including INR and PTT levels, and platelet counts in predicting mortality in patients with ED with suspected infections. [20] These findings may have arise from dysregulation of anticoagulant and procoagulant protein release from hepatocytes, endothelium, and circulating cells. [21] Furthermore, the homeostasis of these systems purportedly shifts to a procoagulant state during inflammatory processes.[22] Our study results are in alignment with the literature, as prolonged PTT and INR values potentially are risk factors for hospitalization and IHM.

Yang et al. reported that lactate levels over $>4$ $\mathrm{mmol} / \mathrm{L}$ were predictive of mortality in septic cancer patients.[16] Similarly, we found a high lactate levels may indicate high risk and can be a predictor for hospitalization and IHM. Yang et al. reported that cardiac troponin I and CK-MB are independent risk factors for mortality in cancer patients, and CK and CK-MB levels, but not brain natriuretic peptide, were associated with mortality.[16] Therefore, we recommend the use of troponin levels as a marker for IHM in cancer patients, provided emergency physicians carefully evaluate the differential diagnoses of the acute coronary syndrome in patients with high troponin levels.

\section{Limitations of the Study}

This study is retrospective, which is subject to the inherent risks of bias associated with this research design, and we could neither evaluate the time between the end of treatment and ED admission nor the patient prognosis post-discharge. 


\section{Conclusion}

Cancer patients undertake a higher number of ED visits. Despite the chronic disease process and relative rarity of oncological emergencies, such as superior vena cava syndrome, emergency physicians should remain updated on the management of cancer patients. The pain was one of the commonest reasons for ED visits; emergency physicians should conduct a thorough differential diagnosis to identify the pain source and provide appropriate pain-control strategies for the patient. Hospitalization and IHM rates are higher in cancer patients compared to non-cancer patients. Therefore, complete blood counts, liver and kidney function tests, coagulation parameters, and lactate levels should be evaluated in these patients concomitantly.

Peer-review: Externally peer-reviewed.

Conflict of Interest: The authors declare that they have no conflict of interest.

Ethics Committee Approval: This retrospective observational study was approved by the Non-Interventional Research Ethics Committee, Gülhane School of Medicine, University of Health Sciences (Approval no. 19/253, June 11, 2019).

Financial Support: The authors declared that this study received no financial support.

Authorship contributions: Concept - S.B., Y.A.A.; Design - S.B., Y.A.A.; Supervision - S.B., Y.A.A.; Funding - None; Materials - None; Data collection and/or processing - S.B., Y.A.A.; Data analysis and/or interpretation - S.B., Y.A.A.; Literature search - S.B., Y.A.A.; Writing - S.B., Y.A.A.; Critical review - S.B., Y.A.A.

\section{References}

1. Miranda B, Vidal SA, Mello MJ, Lima JT, Rêgo JC, Pantaleão MC, et al. Cancer patients, emergencies service and provision of palliative care. Rev Assoc Med Bras (1992) 2016;62:207-11.

2. Rivera DR, Gallicchio L, Brown J, Liu B, Kyriacou DN, Shelburne N. Trends in Adult Cancer-Related Emergency Department Utilization: An Analysis of Data From the Nationwide Emergency Department Sample. JAMA Oncol 2017;3:e172450.

3. Hsu J, Donnelly JP, Moore JX, Meneses K, Williams G, Wang HE. National characteristics of Emergency Department visits by patients with cancer in the United States. Am J Emerg Med 2018;36:2038-43.

4. Lash RS, Bell JF, Reed SC, Poghosyan H, Rodgers J, Kim KK, et al. A Systematic Review of Emergency
Department Use Among Cancer Patients. Cancer Nurs 2017;40:135-44.

5. Yang Z, Yang R, Kwak MJ, Qdaisat A, Lin J, Begley $\mathrm{CE}$, et al. Oncologic emergencies in a cancer center emergency department and in general emergency departments countywide and nationwide. PLoS One 2018;13:e0191658.

6. Ahn S, Lee YS, Lim KS, Lee JL. Emergency department cancer unit and management of oncologic emergencies: experience in Asan Medical Center. Support Care Cancer 2012;20:2205-10.

7. Sadik M, Ozlem K, Huseyin M, AliAyberk B, Ahmet $\mathrm{S}$, Ozgur O. Attributes of cancer patients admitted to the emergency department in one year. World J Emerg Med 2014;5:85-90.

8. Yates M, Barrett A. Oncological emergency admissions to the Norfolk and Norwich University Hospital: an audit of current arrangements and patient satisfaction. Clin Oncol (R Coll Radiol) 2009;21:226-33.

9. Erimşah ME, Yaka E, Yilmaz S, Kama A, Pekdemir M. Inter-rater reliability and validity of the Ministry of Health of Turkey's mandatory emergency triage instrument. Emerg Med Australas 2015;27:210-5.

10. Amado JP, Vasquez R, Huari RW, Sucari AS, Oscanoa TJ. Patients with End-stage Oncologic and Nononcologic Disease in Emergency Service of an Urban Tertiary Hospital. Indian J Palliat Care 2018;24:25-7.

11. Nafsi T, Russell R, Reid CM, Rizvi SM. Audit of deaths less than a week after admission through an emergency department: how accurate was the ED diagnosis and were any deaths preventable?. Emerg Med J 2007;24:691-5.

12. Brown J, Grudzen C, Kyriacou DN, Obermeyer Z, Quest T, Rivera D, et al. The Emergency Care of Patients With Cancer: Setting the Research Agenda. Ann Emerg Med 2016;68:706-11.

13. Wulaningsih W, Holmberg L, Ng T, Rohrmann S, Van Hemelrijck M. Serum leptin, C-reactive protein, and cancer mortality in the NHANES III. Cancer Med 2016;5:120-8.

14. Hope C, Robertshaw A, Cheung KL, Idris I, English E. Relationship between $\mathrm{HbAlc}$ and cancer in people with or without diabetes: a systematic review. Diabet Med 2016;33:1013-25.

15. Boursi B, Giantonio BJ, Lewis JD, Haynes K, Mamtani R, Yang YX. Serum glucose and hemoglobin A1C levels at cancer diagnosis and disease outcome. Eur J Cancer 2016;59:90-8.

16. Yang Z, Qdaisat A, Hu Z, Wagar EA, Reyes-Gibby C, Meng QH, et al. Cardiac Troponin Is a Predictor of Septic Shock Mortality in Cancer Patients in an Emergency Department: A Retrospective Cohort Study. PLoS One 2016;11:e0153492. 
17. Oh CM, Won YJ, Cho H, Lee JK, Park BY, Jun JK, et al. Alanine aminotransferase and gamma-glutamyl transferase have different dose-response relationships with risk of mortality by age. Liver Int 2016;36:126-35.

18. Wulaningsih W, Holmberg L, Garmo H, Malmstrom H, Lambe M, Hammar N, et al. Serum lactate dehydrogenase and survival following cancer diagnosis. $\mathrm{Br}$ J Cancer 2015;113:1389-96.

19. Castle J, Mazmudar A, Bentrem D. Preoperative coagulation abnormalities as a risk factor for ad- verse events after pancreas surgery. J Surg Oncol 2018;117:1305-11.

20. Fischer CM, Yano K, Aird WC, Shapiro NI. Abnormal coagulation tests obtained in the emergency department are associated with mortality in patients with suspected infection. J Emerg Med 2012;42:127-32.

21. Aird WC. The hematologic system as a marker of organ dysfunction in sepsis. Mayo Clin Proc 2003;78:869-81.

22. Esmon CT. The interactions between inflammation and coagulation. Br J Haematol 2005;131:417-30. 